\title{
On the Detection of Regions-of-Interest in Dynamic Contrast-Enhanced MRI
}

\author{
David Raba, Marta Peracaula, Robert Martí, and Joan Martí \\ Institut d'Informàtica i Aplicacions \\ Campus Montilivi 17071 Ed. P-IV, University of Girona \\ $\{$ draba, martapb, marly, joanm\}@eia.udg.es
}

\begin{abstract}
Multivariate imaging technologies such as Dynamic Contrast-Enhanced Magnetic Resonance Imaging (DCE-MRI) have recently gained an important attention as it improves tumour detection. Modelling of contrast media uptake and washout kinetic parameters which are closely related to physiological and anatomical features helps to diagnose and detect a possible cancer. One issue that does not generally receive much attention is the process of detecting regions of interest (ROIs). An automatic region-of-interest (ROI) selection method is presented to avoid the time consuming and subjective task of manual ROI selection, which significantly affects reproducibility and accuracy of measurements.
\end{abstract}

\section{Background}

Among the different types of cancer, breast cancer has one of the largest incidences in the women population and is one of the most common causes of death in occidental countries. X-ray imaging is the most common modality used for the detection of breast cancer. Although in general it provides sufficient information, in some cases it is not conclusive and additional modalities which provide complementary information need to be used, such as magnetic resonance (MRI) and ultrasound (US) images.

DCE-MRI is based on capturing tissue magnetic properties during a short time period when a contrast enhancement agent has been injected to the

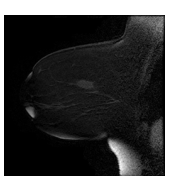

$t_{1}$

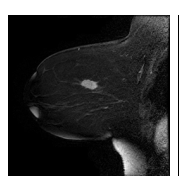

$t_{2}$

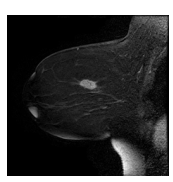

$t_{3}$

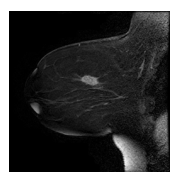

$t_{4}$

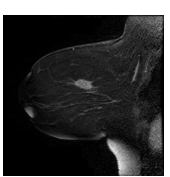

$t_{5}$

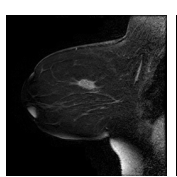

$t_{6}$

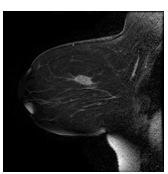

$t_{7}$

Fig. 1. Sample of temporal study with MR mammography, where $t_{1}$ is the precontrast selected slice from whole study and $t_{2}-t_{7}$ are the six post-contrast slices. While parenchyma tissue remains unaffected by the contrast agent, the intensity of the suspicious regions shows an enhancement. 
patient. The increase in intensity throughout the DCE-MRI sequence is a nonlinear function of the uptake of a contrast agent (typically Gadolinium DTPA). Several studies point out DCE-MRI as a valuable tool to discriminate between benign and malign breast lesions [610]. Normal and abnormal tissue will respond differently to the amount of contrast uptake as it is shown in Figure 1 . This contrast differences allows a better visualisation of many different types of tissue abnormalities and disease processes.

A common study for DCE-MRI data consists in looking for breast areas with rapid and strong enhancement of the intensity signal (enhanced by the contrast agent), followed by a quick descent. In terms of contrast uptake-washout behaviour, commonly used approaches for selecting the ROI under evaluation consist of manually outlining the suspicious area. This ROI is within the area of highest contrast agent uptake. Since manually choosing this ROI is more likely to be affected by subjective decisions, it is commonly accepted that inter-observer and intra-observer variability should be minimised hence, reducing reproducibility errors [6. The kinetic curve is formed by calculating the average enhancement over a ROI at all time steps, where the enhancement is defined as the percentage of signal intensity increase relative to the pre-contrast signal intensity as proposed in the study by Kuhl et al. [7] (see Figure 2).

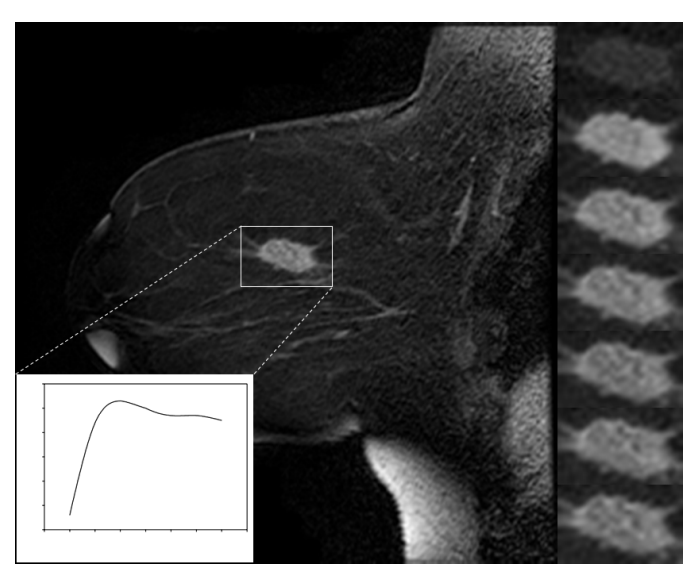

(a)

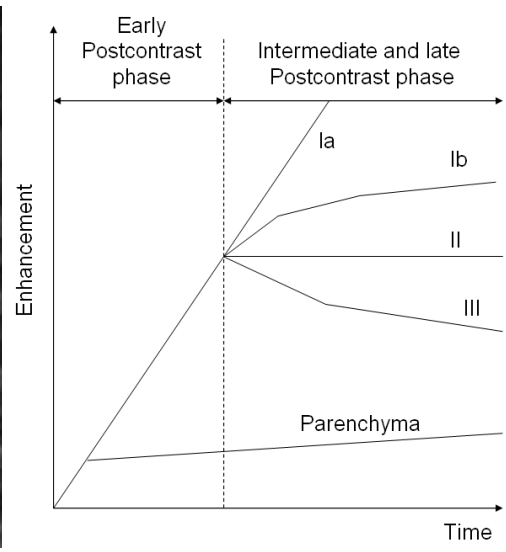

(b)

Fig. 2. Sample of kinetic behaviour where (a) shows the kinetic profile behaviour on manually selected ROI along seven time steps and (b) the typical signal enhancement curves [7] that classify the different profiles as benign tissue (Ia-Ib), suspicious tissue (II), malign tissue (III), and parenchyma response. All curves start with pre-contrast signal within the ROI.

Efforts done in DCE-MRI data analysis include two main strategies, one of them is based on conventional pattern recognition techniques [2] by studying the shape of the extracted profile looking for patterns on the shape using artificial neural networks, support vector machines, or other classification approaches. On 
the other hand, the uptake-washout behaviour of abnormal tissue can also be studied by using pharmacokinetic models which try to describe the time varying distribution of contrast agent during the exchange between blood plasma and extracellular space compartments [1]. In addition the mixture of both strategies has also obtained good results [8]. Mainly these works aim to distinguish between malign or benign ROI, not focusing on obtaining valid ROIs from the whole volume, whereas the goal of our work is to develop an automatic method to detect suspicious regions in DCE-MRI. This localisation of ROI candidates is performed depending on the kinetic and morphological parameters of the kinetic profile.

Briefly outlining the structure of the paper, Section 2 presents our method while Section 3 shows evaluation results. The paper ends with conclusions and further work.

\section{Materials and Methods}

We have evaluated three subjects with DCE-MRI study (GE, Signa LX 1.5T, both breasts, fat saturation, coronal, $512 x 320$ matrix, $1.5 \mathrm{~mm}$ slice thickness). One pre- and six post-contrast one minute series were obtained. In DCE-MRI, the injection of a contrast agent has the property of enhancing highly vascularised regions which is characteristic of malignant regions. In those studies, the patient movement is common as MR acquisition is a relatively long procedure (about 10 minutes). Hence, image registration is a key processing step to improve diagnosing results, correcting slight movements of the patient during the imaging process. Registration has generally been applied to dynamic sequences corresponding a pre-contrast volume to a volume after a contrast agent has been injected. Acquired volumes were automatically registered using $C A D$ Stream ${ }^{T M}$ software.

The method consists of four steps: optimal enhancement localisation, breast tissue segmentation, feature extraction and kinetic curve model identification which are detailed in next sections.

\subsection{Optimal Enhancement Localisation}

Even within a well manually outlined ROI, the shape of kinetic curve extracted from different points within the same ROI presents a high inhomogeneity [2], thereby looking for ROIs with desirable kinetic behaviour (rapid enhancement and posterior washout curve) within the volume obtains a considerable amount of false positives (FP). In order to minimise the number of FP, we propose a novel method to drive the search of ROIs.

An exploration consists of seven volumes $\left(V_{i}\right.$ with $\left.i \in(1 . .7)\right)$, one pre-contrast and six post-contrast acquisitions. We propose the use of a maximum intensity projection (MIP) using the seven volumes to produce a Key-volume that captures the essential enhancement of the whole DCE-MRI exploration. Thus, within the Key-volume each Key-slice $S_{i, j}$ is calculated as the maximum intensity value along the projecting ray trough the stack of images using the $j$-th slice taken from each $V_{i}$ volume. 
Once the Key-volume has been obtained, next step consists of segmenting the key-volume to obtain: (1) a breast segmentation into background and breast volume and (2) a set of regions of interest within the breast. For this task, an unsupervised image segmentation is desirable avoiding the tedious and error prone task of manually segmenting the images. Next section presents our proposal in this direction.

\subsection{Breast Tissue Segmentation}

Certainly, a global segmentation is required since MR breast images show not only breast regions, but also the chest area and non-homogeneous background. Thus, the MR volume is split into breast and non-breast regions. The algorithm used is based on simple morphological filters (two openings and one dilation filtering). Next, a threshold is used to extract the breast region from the background. The value of this threshold is determined using the minimum value between the first two most important peaks, which are the peaks of the background and the breast tissue. Subsequently, a Connected Component Labeling algorithm is used in order to recover the largest area, merging the smaller regions that belong to the breast. The chest area has also been extracted from the image by using the profile segmentation of MRI slice between the two breasts, in order to remove the artifacts from the hearth and other areas not related to the breast.

Once the breast profile is detected, a step forward is to identify the different breast tissue regions. Our internal tissue segmentation approach is based on the Expectation-Maximization algorithm (EM) on a mixture of Gaussians [4]. The EM approach allows to assign each pattern to belong to a specific cluster in a Gaussian Mixture model. In this probabilistic model, each pattern is characterised by a set of mixtures of Gaussian:

$$
p\left(x_{i} ; M\right)=\sum_{m=1}^{M} \pi_{m} g_{m}\left(x_{i}\right)
$$

where $g_{m}$ is a Gaussian distribution and $\pi_{m}$ a prior distribution $\left(\sum_{m} \pi_{m}=1\right)$ and $m$ is the number of mixtures, the different tissue types in our case. The model parameters and cluster membership are determined by maximizing the log-likelihood estimator:

$$
l(M)=\sum_{i} \log \left(p\left(x_{i} ; M\right)\right)
$$

This second step is efficiently done using the Expectation Maximization algorithm 4]. Segmentation process is carried out using 3D intensity data from the previously obtained Key-volume.

\subsection{Feature Extraction}

In order to detect candidate valid ROI we focus on detecting quickly and strongly enhanced regions. These valid ROIs are conceived as possible malignant regions. Therefore, each region obtained after the segmentation step, represents a 
ROI candidate. Nevertheless, we have to deal with wide range of regions sizes. Thereby, assuming that the possible lesion is a candidate ROI and that small regions are more susceptible to be a lesion, a uniform number of samples is obtained for each segmented region. Hence, $N$ points randomly uniformly sampled, without replacement, are selected as seeds from the values in each region. A kinetic curve is obtained by averaging over a $w \times w$ window around the seed.

Additionally, five features are extracted from each kinetic curve to depict maximum contrast enhancement $\left(F_{n 1}\right)$, time to peak $\left(F_{n 2}\right)$, uptake rate $\left(F_{n 3}\right)$, washout rate $\left(F_{n 4}\right)$ [2] and curve angles $\left(F_{n 5}\right)$. The intensity signal of each sample is represented by $S_{t}$ at time step $t(1,2 \ldots, T), S^{*}$ represents the maximum of the T signal intensity values and $p$ is the time point of $S^{*}$. Thus,

$$
S^{*}=\max _{t=1,2, \ldots T} S_{t}, \quad p=\arg \max _{t=1,2, \ldots T} S_{t},
$$

and the four features are defined as,

$$
\begin{gathered}
F_{n 1}=\left(S^{*}-S_{1}\right) / S_{1}, \quad F_{n 2}=p, \quad F_{n 3}=\frac{F_{n 1}}{F_{n 2}}, \quad F_{n 3}=\frac{F_{n 1}}{F_{n 2}} \\
F_{n 4}=\left\{\begin{array}{ll}
\frac{S^{*}-S_{T}}{S_{1}(T-p)} & \text { ifp } \neq T, \\
0 & \text { ifp }=T .
\end{array}, \quad F_{n 5}=\arctan \left(S_{t+1}, S_{t}\right), t(1,2 \ldots, T-1)\right.
\end{gathered}
$$

\subsection{Kinetic Curve Model Identification}

With the feature extraction process a set of features including the uptake profiles are obtained, which should be grouped into three main behaviours: parenchyma, benign or suspicious/malignant. In order to cluster this data we have used a partitional clustering algorithm such as K-Means. It aims to minimise the total within-cluster dispersion, namely

$$
e^{2}(K)=\sum_{k=1}^{K} \sum_{i \in C_{k}}\left\|x_{i}-c_{k}\right\|^{2}
$$

where $c_{k}$ is the centroid of cluster $C_{k}$. K-means clustering is applied by using the set of automatically extracted profiles. An unweighted city-block distance metric is used to evaluate the distance between two profiles. Defined on $\mathbb{R}^{n}$, it is

$$
d(a, b)=\sum_{i=1}^{n}\left|b_{i}-a_{i}\right|
$$

where $a$ and $b$ are vectors in $\mathbb{R}^{n}$ with $a=\left(a_{1}, \ldots, a_{n}\right)$ and $a=\left(b_{1}, \ldots, b_{n}\right)$.

Once the profiles are clustered, we calculate a distance (Eq. 7) within the cluster to assign a probability of belonging to that class. An expert radiologist labels each cluster as being normal, benign or malign. As a result, we obtain for each region a classification into normal, benign or malign and the profile membership probability to its class. Thus, regions with higher probabilities within the suspicious class will be reported as final ROIs. 


\section{$3 \quad$ Evaluation and Results}

The evaluation of the automatic and manual ROI detection is presented using confusion matrices [5] and a leave-one-out scheme over a k-Nearest Neighbours classifier algorithm. In the leave-one-out methodology, each ROI is classified using a model trained with the rest of ROIs, and this procedure is repeated until all ROIs have been used as a query image.

The k-Nearest Neighbours classifier 5] (kNN) consists of classifying a nonclassified vector into the $k$ most similar vectors found in the training set. Because $\mathrm{kNN}$ is based on distances between sample points in feature space, features need to be re-scaled to avoid that some features are weighted much more than others biasing the results. Hence, all features have been normalised to unit variance and zero mean. For the $\mathrm{kNN}$ classifier, the membership value of a class is different from zero if there is at least one neighbour (of $k$ possible neighbours) belonging to this class. The membership value for each class will be the sum of the inverse Euclidean distances among each neighbour of the class and the pattern. A final unit normalisation between all the membership values is required.

To evaluate our approach we have used a DCE-MRI from three patients. Parenchyma, benign and suspicious/malign tissue types are present in this images and manually sampled by an expert providing a total of 106 samples. Program execution is done using the following parameters: Key-volume segmentation into four classes and automatic sample labeling using $\mathrm{K}$-Means with $\mathrm{K}$ value fixed depending on the desired number of classes. Additionally, each confusion matrix include the kappa $(\kappa)$ coefficient [3]. This is used as a means of estimating agreement in categorical data, and is computed as:

$$
\kappa=\frac{P(D)-P(E)}{1-P(E)}
$$

where $P(D)$ is the proportion of times the model values was equal to the actual value (the diagonal terms) and $P(E)$ is the expected proportion by chance. A $\kappa$ coefficient equal to one means a statistically perfect model whereas a value equal to zero means every model value was different from the actual value. Table 1 shows a commonly used interpretation of the various $\kappa$.

Table 2(b-d) shows classification results for the proposed method, (A-kNN) the k-Means profile clustering and the subsequent classification into three and two classes. Comparison with manual profile labelling (M-kNN) is also provided (Table 2(a-b)) in order to evaluate the benefits of using an automatic clustering such as k-Means.

In order to compare classification results between normal and automatic clustered profiles, we must take the possible clustering disagreement into account. That is, different ROIs have different labels and therefore classification could have been using slightly different data. Nevertheless, the labelling agreement has been quantified as being around $77 \%$, which is fairly acceptable. Moreover this error is gathered between parenchyma and normal classes, remaining the suspicious class quite unaffected. Keeping this issue in mind classification results can 
Table 1. Common interpretation of the various kappa values

\begin{tabular}{c|ccccc} 
Agreement & Poor Slight & Fair & Moderate Substantial Almost Perfect \\
\hline$\kappa$ & $<0[0,0.20][0.21,0.40]$ & {$[0.41,0.60]$} & {$[0.61,0.80]$} & {$[0.81,1.00]$}
\end{tabular}

be compared drawing interesting conclusions. Although more data would be desirable, classification results using automatic clustering seem to have a positive impact in reducing the number of False positives (classification says is suspicious when is not) while keeping similar True positive values. This is specially evident for the two-class classification problem. For instance, slightly better result are obtained with automatically labeled data $(\mathrm{A}-\mathrm{kNN})$ in comparison with manually labeled data $(\kappa=0.93$ vs $\kappa=0.89)$ which can be due to its automatically clustered origin. Although these results seem to be promising, further improvements and testing on larger database will show the feasibility of our approach for providing robust ROI detection.

Table 2. Confusion matrices of the kNN classifier. While (a) show results obtained using manually labelled data $(\kappa=0.91)$ and (b) automatically labeled data $(\kappa=0.90)$ taking into account three classes, (c) presents the results using a simplification into two classes (Parenchyma-Normal vs Suspicious, using manually labelled data $(\kappa=0.89)$ and (d) automatically labelled $(\kappa=0.93)$.

\begin{tabular}{|c|c|c|c|c|}
\hline & \multicolumn{3}{|c|}{ M-kNN 3 classes } \\
\hline & & Parenchyma & Normal & Suspicious \\
\hline \multirow{3}{*}{ 皇 } & Parenchyma & 47 & 1 & 2 \\
\hline & Normal & 1 & 36 & 1 \\
\hline & Suspicious & 0 & 1 & 17 \\
\hline
\end{tabular}

(a)

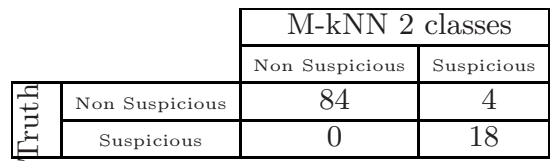

(c)

\begin{tabular}{|c|c|c|c|c|}
\hline & \multicolumn{3}{|c|}{ A-kNN 3 classes } \\
\hline & & Parenchyma & Normal & Suspicious \\
\hline \multirow{3}{*}{ 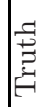 } & Parenchyma & 44 & 2 & 0 \\
\hline & Normal & 1 & 42 & 0 \\
\hline & Suspicious & $\overline{0}$ & 3 & 14 \\
\hline
\end{tabular}

(b)

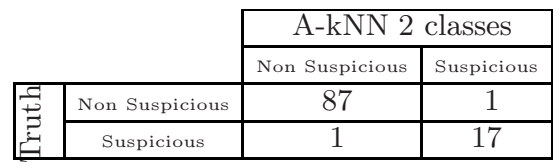

(d)

\section{Conclusions}

An automatic method to detect suspicious area in Dynamic Contrast Enhancement MRI data have been presented in this paper. Besides, a set of discriminant features have been tested using a kNN classifier obtaining satisfactory results compared to manually labelled data. Although the present work has been based on a reduced set of cases, promising results have been obtained. However, this needs to be tested clinically far more thoroughly than we have been able to do to date. The fact that the training of the system is based on direct-image data, not 
depending on an signal modelling step means that the method can be adapted to other imaging protocols and different imaging location like prostate, liver or brain imaging.

Acknowledgments. Thanks to Dr. Joan Carles Vilanova who has provided the image sources. This work has been supported by Spanish CICYT project (TIN2006-08035).

\section{References}

1. Armitage, P., Behrenbruch, C., Brady, M., Moore, N.: Extracting and visualizing physiological parameters using dynamic contrast-enhanced magnetic resonance imaging of the breast. Medical Image Analysis 9, 315-329 (2006)

2. Chen, W., Giger, L., Bick, U., Newstead, G.M.: Automatic identification and classification of characteristic kinetic curves of breast lesions on DCE-MRI. Med. Phys. 33(8), 2878-2887 (2006)

3. Cohen, J.: A coefficient of agreement for nominal scales. Educational and Psicological Measurement 20, 27-46 (1960)

4. Dempster, A.P., Laird, N.M., Rubin, D.B.: Maximum likelihood from incomplete data via the EM algorithm. Journal of the Royal Statistical Society, Series B. 39(1), 1-38 (1977)

5. Duda, R.O., Hart, P.E., Stork, D.G.: Pattern Classification, 2nd edn. John Wiley \& Sons, New York (2001)

6. Galbraith, S., BChir, M.: Antivascular cancer treatments: Imaging biomarkers in pharmaceutical drug development. B. Journal of Radiology 76, 83-86 (2003)

7. Kuhl, C.K., Mielcareck, P., Klaschik, S., Leutner, C., Wardelmann, E., Gieseke, J., Schild, H.H.: Dynamic breast MR imaging: Are signal intensity time course data useful for differential diagnosis of enhancing lesion? Radiology 211, 101-110 (1999)

8. Lucht, R.E.A., Knopp, M.V., Brix, G.: Classification of signal-time curves from dynamic MR mammography by neural networks. Magnetic Resonance Imaging 19(1), $51-57(2000)$

9. Padhani, A.R.: Dynamic Contrast-Enhanced MRI in Clinical Oncology: Current Status and Future Directions. Journal of MRI 16, 407-422 (2002)

10. Szabó, B.K., Aspelin, P., Wiberg, M.K., Boné, B.: Dynamic Mr Imaging Of The Breast: Analysis of kinetic and morphologic diagnostic criteria. Acta Radiologica 44(8), 379-386 (2003) 